\title{
REGULARIZED PERIMETER FOR TOPOLOGY OPTIMIZATION
}

\author{
SAMUEL AMSTUTZ
}

\begin{abstract}
The perimeter functional is known to oppose serious difficulties when it has to be handled within a topology optimization procedure. In this paper, a regularized perimeter functional $\mathrm{Per}_{\varepsilon}$, defined for $2 d$ and $3 d$ domains, is introduced. On one hand, the convergence of Per $_{\varepsilon}$ to the exact perimeter when $\varepsilon$ tends to zero is proved. On the other hand, the topological differentiability of $\mathrm{Per}_{\varepsilon}$ for $\varepsilon>0$ is analyzed. These features lead to the design of a topology optimization algorithm suitable for perimeter dependent objective functionals. Some numerical results on academic problems illustrate the method.
\end{abstract}

\section{INTRODUCTION}

Topology optimization problems are known to be generally ill-posed, in the sense that they possess no global minimizers. Typically, this property stems from the fact that the minimizing sequences have more and more complex topologies, without ever converging to a domain in any appropriate way $[2,13]$. Therefore, relaxation methods are often used $[1,7,9]$, but the binary nature of the problem is then lost. A totally different approach is to impose geometrical constraints that limit the complexity of the obtained topologies. In this framework, a classical technique is to incorporate in the cost function a penalization by the perimeter. In many important cases, the resulting problem can be proved to be well-posed $[3,8,13]$. The control of the perimeter of domains with variable topology appears also in image processing, when considering the Mumford-Shah functional [16], for instance.

However, a major drawback of the perimeter functional is that it is not easy to handle numerically as soon as one wants to perform topology changes. To illustrate this claim, let us consider the creation of a hole $\omega$ inside a larger domain $\Omega$ seen as the current design domain in an iterative process. Then the variation of the perimeter is given by $\operatorname{Per}(\Omega \backslash \bar{\omega})-\operatorname{Per}(\Omega)=\operatorname{Per}(\omega)$. In contrast, the variation of the volume is $|\Omega \backslash \bar{\omega}|-|\Omega|=|\omega|$. In fact, the traditional shape functionals, like the compliance, also admit a first variation proportional to $|\omega|$, at least when Neumann boundary conditions are prescribed on $\partial \omega[4,10,19]$. This difference of order of magnitude creates an incompatibility in the numerical treatment of the perimeter in association with other shape functionals. To circumvent this difficulty, a two step algorithm is used in [14]: a "topological" step which does not take into account the perimeter, then a "classical" step based on smooth boundary variation methods. The basic ingredients in each of these steps are the notions of topological and shape derivatives, respectively. More sophisticated approaches, also based on alternating steps, have been proposed in [11, 12].

In this paper, we present a natural way to include the perimeter within a topology optimization procedure. The proposed approach is based on a regularization method: the perimeter $\operatorname{Per}(\Omega)$ is approximated by a functional $\operatorname{Per}_{\varepsilon}(\Omega)$ well-suited for topology optimization, then $\varepsilon$ is driven to zero for which the exact perimeter is retrieved. Let us enter a little more into details. Let $\Omega$ be an open and bounded subset of $\mathbb{R}^{N}, N \in\{2,3\}$, with $\mathcal{C}^{2}$ boundary $\partial \Omega$. We denote by $u$ the characteristic function of $\Omega$, i.e., $u(x)=1$ if $x \in \Omega, u(x)=0$ if $x \in \mathbb{R}^{N} \backslash \Omega$. For a fixed $m \in \mathbb{N}^{\star}$ and any $\varepsilon>0$ we consider the (weak) solution $u_{\varepsilon} \in H^{m}\left(\mathbb{R}^{N}\right)$ of

$$
\varepsilon^{2 m}(-\Delta)^{m} u_{\varepsilon}+u_{\varepsilon}=u .
$$

Then we define the quantity

$$
E_{\varepsilon}(\Omega):=\left\|u-u_{\varepsilon}\right\|_{L^{2}\left(\mathbb{R}^{N}\right)}^{2}=\int_{\mathbb{R}^{N}}\left(u-u_{\varepsilon}\right)^{2} d x .
$$

We shall see that the asymptotic behavior of $E_{\varepsilon}(\Omega)$ when $\varepsilon$ goes to zero is directly related to the perimeter of $\Omega$. Before giving a precise statement, let us specify some notation. We denote by $\langle.,$. the canonical scalar product of $\mathbb{R}^{N}$, and by $|$.$| the associated norm. For complex vectors, the same$

Key words and phrases. topology optimization, perimeter, topological derivative, level set. 
notation is kept for the Hermitian scalar product of $\mathbb{C}^{N}$ and its norm, while complex conjugacy is denoted by a bar. The surface measure on $\partial \Omega$ is denoted by $\sigma$. Therefore, the perimeter of $\Omega$ can be defined as

$$
\operatorname{Per}(\Omega)=\sigma(\partial \Omega)=\int_{\partial \Omega} d \sigma
$$

The outward unit normal to $\partial \Omega$ at point $x$ is denoted by $n(x)$. We shall prove the following result.

Theorem 1.1. The following asymptotic expansion holds when $\varepsilon$ goes to zero:

$$
E_{\varepsilon}(\Omega)=\varepsilon \kappa_{m} \operatorname{Per}(\Omega)+O\left(\varepsilon^{\frac{N+4}{N+2}}\right),
$$

where $\kappa_{m}$ is defined by

$$
\kappa_{m}=\frac{1}{\pi} \int_{0}^{\infty} \frac{t^{4 m-2}}{\left(1+t^{2 m}\right)^{2}} d t .
$$

The first values of $\kappa_{m}$ are $\kappa_{1}=1 / 4$ and $\kappa_{2}=3 / 2^{7 / 2}$.

Therefore, we call regularized perimeter the quantity

$$
\operatorname{Per}_{\varepsilon}(\Omega)=\frac{1}{\kappa_{m} \varepsilon} E_{\varepsilon}(\Omega)
$$

which, in consequence of Theorem 1.1, satisfies

$$
\operatorname{Per}_{\varepsilon}(\Omega)=\operatorname{Per}(\Omega)+O\left(\varepsilon^{\frac{2}{N+2}}\right) .
$$

Theorem 1.1 is proved in Section 2. In Section 3, the result is extended to a boundary value problem, where (1.1) is complemented by a Neumann boundary condition on the border of a bounded domain $D$ containing $\Omega$. In Section 4, the sensitivity of the functional $\operatorname{Per}_{\varepsilon}$ to topological perturbations is analyzed. Then, in Section 5, we show how these results lead to a topology optimization algorithm dedicated to perimeter dependent objective functionals. Finally, some numerical experiments on a simple model problem are reported in Section 6 .

\section{Asymptotic EXPANSION OF the REgUlarized FUnCTIONAL}

This section is devoted to the proof of Theorem 1.1. Our approach relies on the Fourier transform, for which we adopt the definition

$$
\forall f \in L^{1}\left(\mathbb{R}^{N}\right), \quad \hat{f}(\xi)=(2 \pi)^{-N / 2} \int_{\mathbb{R}^{N}} e^{-i\langle x, \xi\rangle} f(x) d x .
$$

For a detailed exposition of the Fourier transform's properties, we refer, e.g., to [15].

2.1. Reformulation in the frequency domain. Passing to the Fourier transform in (1.1) yields

$$
\varepsilon^{2 m}|\xi|^{2 m} \hat{u}_{\varepsilon}(\xi)+\hat{u}_{\varepsilon}(\xi)=\hat{u}(\xi),
$$

from which we derive

Next, by Parseval's equality, we obtain

$$
\hat{u}_{\varepsilon}(\xi)=\frac{\hat{u}(\xi)}{1+(\varepsilon|\xi|)^{2 m}} .
$$

$$
E_{\varepsilon}(\Omega)=\left\|\hat{u}-\hat{u}_{\varepsilon}\right\|_{L^{2}\left(\mathbb{R}^{N}\right)}^{2}=\int_{\mathbb{R}^{N}}\left(\frac{(\varepsilon|\xi|)^{2 m}}{1+(\varepsilon|\xi|)^{2 m}}\right)^{2}|\hat{u}(\xi)|^{2} d \xi
$$

The change of variable $\zeta=\varepsilon \xi$ results in

$$
E_{\varepsilon}(\Omega)=\varepsilon^{-N} \int_{\mathbb{R}^{N}} \frac{|\zeta|^{4 m}}{\left(1+|\zeta|^{2 m}\right)^{2}}\left|\hat{u}\left(\varepsilon^{-1} \zeta\right)\right|^{2} d \zeta
$$

It will turn out to be useful and also interesting on its own to study a generalized version of (2.1). To this aim, for all $k \in \mathbb{N}$, we introduce the linear space

$$
\mathcal{V}_{k}=\left\{\Phi \in \mathcal{C}^{\infty}(\mathbb{R}),\left[t \mapsto t^{k-2}\left(1+t^{2}\right)^{2} \Phi^{(k)}(t)\right] \in L^{\infty}(\mathbb{R})\right\},
$$

endowed with the norm

$$
\|\Phi\|_{\mathcal{V}_{k}}=\left\|t \mapsto t^{k-2}\left(1+t^{2}\right)^{2} \Phi^{(k)}(t)\right\|_{L^{\infty}(\mathbb{R})}=\inf \left\{a \in \mathbb{R},\left|\Phi^{(k)}(t)\right| \leq a \frac{|t|^{2-k}}{\left(1+t^{2}\right)^{2}} \text { a.e. } t \in \mathbb{R}\right\} .
$$


Then, for all $\Phi \in \mathcal{V}_{0}$, we set

$$
T_{\varepsilon}(\Phi)=\varepsilon^{-N} \int_{\mathbb{R}^{N}} \Phi(|\zeta|)|\zeta|^{2}\left|\hat{u}\left(\varepsilon^{-1} \zeta\right)\right|^{2} d \zeta
$$

Since $\hat{u} \in L^{2}\left(\mathbb{R}^{N}\right)$, the above expression makes sense for all $\Phi \in \mathcal{V}_{0}$, and furthermore we have $T_{\varepsilon} \in \mathcal{V}_{0}^{\prime}$, the continuous dual of $\mathcal{V}_{0}$. We also define the linear functional $\tilde{T}_{\varepsilon} \in \mathcal{V}_{0}^{\prime}$ by

and the linear space

$$
\tilde{T}_{\varepsilon}(\Phi)=\frac{\varepsilon}{\pi} \operatorname{Per}(\Omega) \int_{0}^{\infty} \Phi(t) d t
$$

endowed with the norm

$$
\mathcal{V}=\bigcap_{k=0}^{N+1} \mathcal{V}_{k}
$$

We shall prove the following result.

$$
\|\Phi\|_{\mathcal{V}}=\max \left\{\|\Phi\|_{\mathcal{V}_{k}}, k=0, \ldots, N+1\right\}
$$

Theorem 2.1. There exists $c>0$ such that, for all $\Phi \in \mathcal{V}$ and all $\varepsilon$ sufficiently small,

$$
\left|T_{\varepsilon}(\Phi)-\tilde{T}_{\varepsilon}(\Phi)\right| \leq c \varepsilon^{\frac{N+4}{N+2}}\|\Phi\|_{\mathcal{V}} .
$$

Then Theorem 1.1 follows at once from Theorem 2.1 by choosing

$$
\Phi(t)=\frac{t^{4 m-2}}{\left(1+t^{2 m}\right)^{2}} .
$$

We only have to check that this function belongs to $\mathcal{V}$. To do so we set $G_{k}(t)=t^{2-k} /\left(1+t^{2}\right)^{2}$. We remark that $\Phi^{(k)} / G_{k}$ is a rational function of degree 0 , hence it will be bounded as soon as it has no pole on the real line. Immediate calculations provide

$$
\begin{aligned}
\frac{\Phi(t)}{G_{0}(t)} & =\left[t^{2 m-2} \frac{1+t^{2}}{1+t^{2 m}}\right]^{2}, \\
\frac{\Phi^{\prime}(t)}{G_{1}(t)} & =(4 m-2)\left[t^{2 m-2} \frac{1+t^{2}}{1+t^{2 m}}\right]^{2}-4 m t^{6 m-4} \frac{\left(1+t^{2}\right)^{2}}{\left(1+t^{2 m}\right)^{3}}, \\
\forall k \geq 2, \quad \frac{\Phi^{(k)}(t)}{G_{k}(t)} & =\Phi^{(k)}(t) t^{k-2}\left(1+t^{2}\right)^{2} .
\end{aligned}
$$

Obviously the above rational functions have no real poles for any $m \geq 1$.

The rest of this section is devoted to the proof of Theorem 2.1. Throughout, the letter $c$ will be used to denote any positive constant independent of $\varepsilon$ and $\Phi$. For the reader's convenience the proof is divided into three parts.

2.2. Derivation of the leading term. At first, we assume that $\Phi \in \mathcal{C}_{0}^{\infty}(\mathbb{R})$, the set of functions of class $\mathcal{C}^{\infty}$ on $\mathbb{R}$ with compact support. By definition we have

$$
|\xi|^{2} \hat{u}(\xi)=(2 \pi)^{-N / 2}|\xi|^{2} \int_{\Omega} e^{-i\langle x, \xi\rangle} d x=(2 \pi)^{-N / 2} \int_{\Omega} \operatorname{div}_{x}\left(i e^{-i\langle x, \xi\rangle} \xi\right) d x
$$

which, by the divergence formula and setting $e_{\xi}=\xi /|\xi|$, yields

$$
|\xi| \hat{u}(\xi)=(2 \pi)^{-N / 2} i \int_{\partial \Omega} e^{-i\langle x, \xi\rangle}\left\langle e_{\xi}, n(x)\right\rangle d \sigma(x) .
$$

On writing $|\hat{u}(\xi)|^{2}=\hat{u}(\xi) \overline{\hat{u}(\xi)}$ we obtain from $(2.3)$

$$
|\xi|^{2}|\hat{u}(\xi)|^{2}=(2 \pi)^{-N} \int_{\partial \Omega \times \partial \Omega} e^{-i\langle x-y, \xi\rangle}\left\langle e_{\xi}, n(x)\right\rangle\left\langle e_{\xi}, n(y)\right\rangle d \sigma(x) d \sigma(y) .
$$

Plugging this expression into (2.2) entails

$$
T_{\varepsilon}(\Phi)=(2 \pi)^{-N} \varepsilon^{2-N} \int_{\mathbb{R}^{N}} \Phi(|\zeta|)\left[\int_{\partial \Omega \times \partial \Omega} e^{i \varepsilon^{-1}\langle y-x, \zeta\rangle}\left\langle e_{\zeta}, n(x)\right\rangle\left\langle e_{\zeta}, n(y)\right\rangle d \sigma(x) d \sigma(y)\right] d \zeta .
$$

By Fubini's theorem, this can be reordered as

$$
T_{\varepsilon}(\Phi)=(2 \pi)^{-N} \varepsilon^{2-N} \int_{\partial \Omega}\left\langle\int_{\partial \Omega}\left(\int_{\mathbb{R}^{N}} e^{i \varepsilon^{-1}\langle y-x, \zeta\rangle} \Phi(|\zeta|) e_{\zeta} \otimes e_{\zeta} d \zeta\right) n(y) d \sigma(y), n(x)\right\rangle d \sigma(x) .
$$


Setting

we arrive at

$$
\begin{gathered}
\varphi(z)=(2 \pi)^{-N / 2} \int_{\mathbb{R}^{N}} e^{i\langle z, \zeta\rangle} \Phi(|\zeta|) e_{\zeta} \otimes e_{\zeta} d \zeta, \\
F_{\varepsilon}(x)=\int_{\partial \Omega} \varphi\left(\varepsilon^{-1}(y-x)\right) n(y) d \sigma(y),
\end{gathered}
$$

$$
T_{\varepsilon}(\Phi)=(2 \pi)^{-N / 2} \varepsilon^{2-N} \int_{\partial \Omega}\left\langle F_{\varepsilon}(x), n(x)\right\rangle d \sigma(x) .
$$

We shall now examine the asymptotic behavior of $F_{\varepsilon}(x)$ for a given $x \in \partial \Omega$. Let $\rho>0$ be such that the set $\partial \Omega \cap B(x, 2 \rho)$ can be represented as the graph of a $\mathcal{C}^{2}$ function on an appropriate local Cartesian coordinate system. Note that, by compactness of $\partial \Omega, \rho$ may be chosen independent of $x$. Let $\eta: \mathbb{R}^{N} \rightarrow \mathbb{R}$ be a smooth $\left(\mathcal{C}^{\infty}\right)$ function such that $\eta(z)=1$ if $|z| \leq \rho, 0 \leq \eta(z) \leq 1$ if $\rho \leq|z| \leq 2 \rho$, and $\eta(z)=0$ if $|z| \geq 2 \rho$. We introduce a parameter $\beta \in] 0,1[$ which will be fixed later, and split (2.5) as

$$
F_{\varepsilon}(x)=F_{\varepsilon}^{0}(x)+F_{\varepsilon}^{1}(x),
$$

with

$$
\begin{gathered}
F_{\varepsilon}^{0}(x)=\int_{\partial \Omega} \eta\left(\varepsilon^{-\beta}(y-x)\right) \varphi\left(\varepsilon^{-1}(y-x)\right) n(y) d \sigma(y), \\
F_{\varepsilon}^{1}(x)=\int_{\partial \Omega}\left[1-\eta\left(\varepsilon^{-\beta}(y-x)\right)\right] \varphi\left(\varepsilon^{-1}(y-x)\right) n(y) d \sigma(y) .
\end{gathered}
$$

In the ball $B(x, 2 \rho)$ we parametrize $\partial \Omega$ by

$$
t \in \mathcal{O} \mapsto y(t)=x+R(t, \psi(t)) \in \partial \Omega,
$$

where $\mathcal{O}$ is an open set of $\mathbb{R}^{N-1}$ containing the origin, $R$ is a rotation, and $\psi: \mathcal{O} \rightarrow \mathbb{R}$ is a function of class $\mathcal{C}^{2}$ satisfying

$$
\psi(0)=0, \quad \nabla \psi(0)=0 .
$$

For notational simplicity, we write vectors of $\mathbb{R}^{N}$ indifferently row-wise or column-wise. We subsequently assume that $\varepsilon<1$. Then $\eta\left(\varepsilon^{-\beta}(y-x)\right) \neq 0$ implies $y \in B(x, 2 \rho)$, and we can write

$$
F_{\varepsilon}^{0}(x)=\int_{\mathcal{O}} \eta\left(\varepsilon^{-\beta}(y(t)-x)\right) \varphi\left(\varepsilon^{-1}(y(t)-x)\right) R(-\nabla \psi(t), 1) d t .
$$

Setting

we obtain

$$
\eta_{\varepsilon}(t)= \begin{cases}\eta\left(\varepsilon^{-\beta}(y(t)-x)\right) & \text { if } t \in \mathcal{O} \\ 0 & \text { otherwise }\end{cases}
$$

$$
F_{\varepsilon}^{0}(x)=\int_{\mathbb{R}^{N-1}} \eta_{\varepsilon}(t) \varphi\left(R\left(\varepsilon^{-1} t, \varepsilon^{-1} \psi(t)\right)\right) R(-\nabla \psi(t), 1) d t .
$$

By the definition (2.4), we observe that

$$
\varphi(R z)=R \varphi(z) R^{\star}
$$

with $R^{\star}$ the adjoint of $R$. This entails

$$
F_{\varepsilon}^{0}(x)=R \int_{\mathbb{R}^{N-1}} \eta_{\varepsilon}(t) \varphi\left(\varepsilon^{-1} t, \varepsilon^{-1} \psi(t)\right)(-\nabla \psi(t), 1) d t .
$$

Then, by the change of variable $t=\varepsilon s$, we arrive at

$$
F_{\varepsilon}^{0}(x)=\varepsilon^{N-1} R \int_{\mathbb{R}^{N-1}} \eta_{\varepsilon}(\varepsilon s) \varphi\left(s, \varepsilon^{-1} \psi(\varepsilon s)\right)(-\nabla \psi(\varepsilon s), 1) d s .
$$

Let $e_{N}=(0, \ldots, 0,1)$ be the last vector of the canonical basis of $\mathbb{R}^{N}$. We split $(2.13)$ as

$$
F_{\varepsilon}^{0}(x)=A_{\varepsilon}(x)+B_{\varepsilon}(x)+C_{\varepsilon}(x)+D_{\varepsilon}(x),
$$

with

$$
\begin{gathered}
A_{\varepsilon}(x)=\varepsilon^{N-1} R \int_{\mathbb{R}^{N-1}} \varphi(s, 0) e_{N} d s, \\
B_{\varepsilon}(x)=\varepsilon^{N-1} R \int_{\mathbb{R}^{N-1}}\left[\eta_{\varepsilon}(\varepsilon s)-1\right] \varphi(s, 0) e_{N} d s,
\end{gathered}
$$




$$
\begin{gathered}
C_{\varepsilon}(x)=\varepsilon^{N-1} R \int_{\mathbb{R}^{N-1}} \eta_{\varepsilon}(\varepsilon s)\left[\varphi\left(s, \varepsilon^{-1} \psi(\varepsilon s)\right)-\varphi(s, 0)\right] e_{N} d s, \\
D_{\varepsilon}(x)=\varepsilon^{N-1} R \int_{\mathbb{R}^{N-1}} \eta_{\varepsilon}(\varepsilon s) \varphi\left(s, \varepsilon^{-1} \psi(\varepsilon s)\right)(-\nabla \psi(\varepsilon s), 0) d s .
\end{gathered}
$$

We first focus on the expected leading term $A_{\varepsilon}(x)$. We define for every $\zeta \in \mathbb{R}^{N}$ and $\zeta^{\prime} \in \mathbb{R}^{N-1}$

$$
h(\zeta)=\Phi(|\zeta|) e_{\zeta} \otimes e_{\zeta}, \quad H\left(\zeta^{\prime}\right)=\int_{\mathbb{R}} h\left(\zeta^{\prime}, \zeta_{N}\right) d \zeta_{N} .
$$

Therefore the definition (2.4) is equivalent to $\varphi(z)=\overline{\hat{h}(z)}$. In addition, we have for all $s \in \mathbb{R}^{N-1}$

$$
\hat{H}(s)=(2 \pi)^{-\frac{N-1}{2}} \int_{\mathbb{R}^{N-1}} e^{-i\left\langle s, \zeta^{\prime}\right\rangle} \int_{\mathbb{R}} h\left(\zeta^{\prime}, \zeta_{N}\right) d \zeta_{N} d \zeta^{\prime}=(2 \pi)^{\frac{1}{2}} \hat{h}(s, 0),
$$

thus

It follows that

$$
\varphi(s, 0)=(2 \pi)^{-\frac{1}{2}} \overline{\hat{H}(s)} .
$$

$$
A_{\varepsilon}(x)=(2 \pi)^{-\frac{1}{2}} \varepsilon^{N-1} R\left[\int_{\mathbb{R}^{N-1}} \overline{\hat{H}(s)} d s\right] e_{N} .
$$

Next, the Fourier inversion formula yields

$$
A_{\varepsilon}(x)=(2 \pi)^{\frac{N}{2}-1} \varepsilon^{N-1} R \overline{H(0)} e_{N} .
$$

From

we arrive at

$$
H(0)=\int_{\mathbb{R}} \Phi\left(\left|\zeta_{N}\right|\right) e_{N} \otimes e_{N} d \zeta_{N}=2\left(\int_{0}^{\infty} \Phi(t) d t\right) e_{N} \otimes e_{N},
$$

$$
A_{\varepsilon}(x)=(2 \pi)^{\frac{N}{2}-1} \varepsilon^{N-1} 2\left(\int_{0}^{\infty} \Phi(t) d t\right) R e_{N}=(2 \pi)^{\frac{N}{2}-1} \varepsilon^{N-1} 2\left(\int_{0}^{\infty} \Phi(t) d t\right) n(x) .
$$

The contribution of $A_{\varepsilon}(x)$ in the functional $E_{\varepsilon}(\Omega)$ is then given by

$$
\begin{aligned}
(2 \pi)^{-N / 2} \varepsilon^{2-N} \int_{\partial \Omega}\left\langle A_{\varepsilon}(x), n(x)\right\rangle d \sigma(x) & =(2 \pi)^{-1} \varepsilon 2\left(\int_{0}^{\infty} \Phi(t) d t\right) \int_{\partial \Omega}\langle n(x), n(x)\rangle d \sigma(x) \\
& =\frac{\varepsilon}{\pi}\left(\int_{0}^{\infty} \Phi(t) d t\right) \operatorname{Per}(\Omega)=\tilde{T}_{\varepsilon}(\Phi) .
\end{aligned}
$$

2.3. Estimate of remainders. From (2.6) and (2.21) we find

$$
T_{\varepsilon}(\Phi)-\tilde{T}_{\varepsilon}(\Phi)=(2 \pi)^{-N / 2} \varepsilon^{2-N} \int_{\partial \Omega}\left\langle F_{\varepsilon}(x)-A_{\varepsilon}(x), n(x)\right\rangle d \sigma(x) .
$$

Then using (2.7) and (2.14) we arrive at

$$
T_{\varepsilon}(\Phi)-\tilde{T}_{\varepsilon}(\Phi)=(2 \pi)^{-N / 2} \varepsilon^{2-N} \int_{\partial \Omega}\left\langle F_{\varepsilon}^{1}(x)+B_{\varepsilon}(x)+C_{\varepsilon}(x)+D_{\varepsilon}(x), n(x)\right\rangle d \sigma(x) .
$$

We shall estimate each term of the integrand in (2.22). Beforehand, we shall establish useful estimates for the function $\varphi$ defined by (2.4).

By successive integrations by parts from (2.4), we obtain for each $j \in\{1, \ldots, N\}$ and any $n \in \mathbb{N}$

$$
\varphi(z) z_{j}^{n}=(2 \pi)^{-N / 2} i^{n} \int_{\mathbb{R}^{N}} e^{i\langle z, \zeta\rangle} \frac{\partial^{n}}{\partial \zeta_{j}^{n}}\left(\Phi(|\zeta|) e_{\zeta} \otimes e_{\zeta}\right) d \zeta .
$$

Here, $z_{j}$ and $\zeta_{j}$ stand for the $j-t h$ components of the vectors $z$ and $\zeta$, respectively. The Leibniz formula provides

$$
\frac{\partial^{n}}{\partial \zeta_{j}^{n}}\left(\Phi\left(|\zeta| e_{\zeta} \otimes e_{\zeta}\right)=\frac{\partial}{\partial \zeta_{j}^{n}}\left(\frac{\Phi(|\zeta|)}{|\zeta|^{2}} \zeta \otimes \zeta\right)=\sum_{k=0}^{2}\left(\begin{array}{l}
n \\
k
\end{array}\right) \frac{\partial^{n-k}}{\partial \zeta_{j}^{n-k}}\left(\frac{\Phi(|\zeta|)}{|\zeta|^{2}}\right) \frac{\partial^{k}}{\partial \zeta_{j}^{k}}(\zeta \otimes \zeta) .\right.
$$

By induction, deferred to the appendix, we prove that

$$
\frac{\partial^{q}}{\partial \zeta_{j}^{q}}\left(\frac{\Phi(|\zeta|)}{|\zeta|^{2}}\right)=\frac{1}{|\zeta|^{2 q+2}} \sum_{p=0}^{q} \Phi^{(p)}(|\zeta|) P_{p, q}\left(|\zeta|, \zeta_{j}\right)
$$


where $P_{p, q}$ is a homogeneous polynomial of two variables of degree $p+q$. This entails

$$
\left|\frac{\partial^{q}}{\partial \zeta_{j}^{q}}\left(\frac{\Phi(|\zeta|)}{|\zeta|^{2}}\right)\right| \leq c_{q} \frac{1}{|\zeta|^{2 q+2}} \sum_{p=0}^{q}\left|\Phi^{(p)}(|\zeta|)\right||\zeta|^{p+q}
$$

for some constants $c_{q}>0$. Using now that, by definition,

$$
\left|\Phi^{(p)}(|\zeta|)\right| \leq\|\Phi\|_{\mathcal{V}_{p}} \frac{|\zeta|^{2-p}}{\left(1+|\zeta|^{2}\right)^{2}},
$$

we obtain

$$
\left|\frac{\partial^{q}}{\partial \zeta_{j}^{q}}\left(\frac{\Phi(|\zeta|)}{|\zeta|^{2}}\right)\right| \leq(q+1) c_{q} \frac{\max \left(\|\Phi\|_{\mathcal{V}_{p}}, p \leq q\right)}{|\zeta|^{q}\left(1+|\zeta|^{2}\right)^{2}} .
$$

Plugging this estimate into (2.24), we get

$$
\begin{aligned}
\mid \frac{\partial^{n}}{\partial \zeta_{j}^{n}}\left(\Phi\left(|\zeta| e_{\zeta} \otimes e_{\zeta}\right) \mid\right. & \leq \sum_{k=0}^{2}\left(\begin{array}{l}
n \\
k
\end{array}\right)(n-k+1) c_{n-k} \frac{\max \left(\|\Phi\|_{\mathcal{V}_{p}}, p \leq n-k\right)}{|\zeta|^{n-k}\left(1+|\zeta|^{2}\right)^{2}}|\zeta|^{2-k} \\
& \leq c_{n} \frac{\max \left(\|\Phi\|_{\left.\mathcal{V}_{p}, p \leq n\right)}\right.}{|\zeta|^{n-2}\left(1+|\zeta|^{2}\right)^{2}},
\end{aligned}
$$

for some other constant $c_{n}>0$. The combination of (2.23) and (2.27) leads to

$$
|\varphi(z) \| z|^{n} \leq(2 \pi)^{-N / 2} c_{n} \max \left(\|\Phi\|_{\mathcal{V}_{p}}, p \leq n\right) \int_{\mathbb{R}^{N}} \frac{d \zeta}{|\zeta|^{n-2}\left(1+|\zeta|^{2}\right)^{2}} .
$$

The integral at the right hand side of the above inequality is finite whenever $N-1 \leq n \leq N+1$. We conclude that

$$
\forall n \in\{N-1, N, N+1\}, \quad|\varphi(z)| \leq c \frac{\max \left(\|\Phi\|_{\mathcal{V}_{p}}, p \leq n\right)}{|z|^{n}} \leq c \frac{\|\Phi\|_{\mathcal{V}}}{|z|^{n}} .
$$

Next we study the partial derivative

$$
\frac{\partial \varphi}{\partial z_{N}}(z)=(2 \pi)^{-N / 2} i \int_{\mathbb{R}^{N}} e^{i\langle z, \zeta\rangle} \zeta_{N} \Phi(|\zeta|) e_{\zeta} \otimes e_{\zeta} d \zeta .
$$

By successive integrations by parts we find

$$
\frac{\partial \varphi}{\partial z_{N}}(z) z_{j}^{n}=(2 \pi)^{-N / 2} i^{n+1} \int_{\mathbb{R}^{N}} e^{i\langle z, \zeta\rangle} \frac{\partial^{n}}{\partial \zeta_{j}^{n}}\left(\zeta_{N} \Phi(|\zeta|) e_{\zeta} \otimes e_{\zeta}\right) d \zeta .
$$

If $j \neq N$ we have obviously

$$
\frac{\partial \varphi}{\partial z_{N}}(z) z_{j}^{n}=(2 \pi)^{-N / 2} i^{n+1} \int_{\mathbb{R}^{N}} e^{i\langle z, \zeta\rangle} \zeta_{N} \frac{\partial^{n}}{\partial \zeta_{j}^{n}}\left(\Phi(|\zeta|) e_{\zeta} \otimes e_{\zeta}\right) d \zeta .
$$

Using (2.27) we obtain

$$
\forall j \neq N, \quad\left|\frac{\partial \varphi}{\partial z_{N}}(z)\right|\left|z_{j}\right|^{n} \leq(2 \pi)^{-N / 2} c_{n} \max \left(\|\Phi\|_{\mathcal{V}_{p}}, p \leq n\right) \int_{\mathbb{R}^{N}} \frac{d \zeta}{|\zeta|^{n-1}\left(1+|\zeta|^{2}\right)^{2}} .
$$

For $j=N$ the Leibniz formula provides

$$
\frac{\partial^{n}}{\partial \zeta_{N}^{n}}\left(\zeta_{N} \Phi(|\zeta|) e_{\zeta} \otimes e_{\zeta}\right)=\zeta_{N} \frac{\partial^{n}}{\partial \zeta_{N}^{n}}\left(\Phi(|\zeta|) e_{\zeta} \otimes e_{\zeta}\right)+n \frac{\partial^{n-1}}{\partial \zeta_{N}^{n-1}}\left(\Phi(|\zeta|) e_{\zeta} \otimes e_{\zeta}\right) .
$$

Then (2.27) yields

$$
\left|\frac{\partial^{n}}{\partial \zeta_{N}^{n}}\left(\zeta_{N} \Phi(|\zeta|) e_{\zeta} \otimes e_{\zeta}\right)\right| \leq(2 \pi)^{-N / 2}\left(c_{n}+n c_{n-1}\right) \frac{\max \left(\|\Phi\|_{\mathcal{V}_{p}}, p \leq n\right)}{|\zeta|^{n-3}\left(1+|\zeta|^{2}\right)^{2}},
$$

which, in view of (2.29), implies

$$
\left|\frac{\partial \varphi}{\partial z_{N}}(z)\right|\left|z_{N}\right|^{n} \leq(2 \pi)^{-N / 2}\left(c_{n}+n c_{n-1}\right) \max \left(\|\Phi\|_{\mathcal{V}_{p}}, p \leq n\right) \int_{\mathbb{R}^{N}} \frac{\left.d \zeta\right|^{n-3}\left(1+|\zeta|^{2}\right)^{2}}{\mid .}
$$


The two integrals at right hand sides of (2.30) and (2.31) are finite if we choose $n=N$. We conclude that

$$
\left|\frac{\partial \varphi}{\partial z_{N}}(z)\right| \leq c \frac{\|\Phi\|_{\mathcal{V}}}{|z|^{N}}
$$

(1) When $\varepsilon^{-\beta}(y-x)$ belongs to the support of $1-\eta$, we have $\varepsilon^{-\beta}|y-x| \geq \rho$, hence, in view of (2.28) for $n=N+1$,

$$
1-\eta\left(\varepsilon^{-\beta}(y-x)\right) \neq 0 \Longrightarrow\left|\varphi\left(\varepsilon^{-1}(y-x)\right)\right| \leq c \frac{\|\Phi\|_{\mathcal{V}}}{\left(\rho \varepsilon^{\beta-1}\right)^{N+1}} .
$$

From (2.9) and the above estimate we infer

$$
\left|F_{\varepsilon}^{1}(x)\right| \leq c \varepsilon^{(1-\beta)(N+1)}\|\Phi\|_{\mathcal{V}} .
$$

(2) From (2.16) we derive

$$
\left|B_{\varepsilon}(x)\right| \leq \varepsilon^{N-1} \int_{\mathbb{R}^{N-1}}\left(1-\eta_{\varepsilon}(\varepsilon s)\right)|\varphi(s, 0)| d s .
$$

In view of (2.10) we have

$$
\forall t \in \mathcal{O}, \quad|y(t)-x|=\sqrt{|t|^{2}+\psi(t)^{2}} .
$$

Yet, using (2.11) and a Taylor-Lagrange expansion, we get

$$
\forall t \in \mathcal{O}, \quad|\psi(t)| \leq c|t|^{2},
$$

hence

$$
\forall t \in \mathcal{O}, \quad|y(t)-x| \leq \sqrt{|t|^{2}+c|t|^{4}} \leq \lambda|t|
$$

for some $\lambda \geq 1$. We deduce that

$$
\forall t \in \mathcal{O}, \quad|t| \leq \frac{\rho}{\lambda} \varepsilon^{\beta} \Longrightarrow \varepsilon^{-\beta}|y(t)-x| \leq \rho \Longrightarrow \eta_{\varepsilon}(t)=1 .
$$

Set $\alpha=\rho / \lambda$, possibly decreased so that $B(0, \alpha) \subset \mathcal{O}$. Thus, for all $t \in \mathbb{R}^{N-1},|t| \leq \alpha \varepsilon^{\beta}$ implies $\eta_{\varepsilon}(t)=1$. Using also (2.28) for $n=N+1$, we arrive at

$$
\begin{aligned}
\left|B_{\varepsilon}(x)\right| & \leq c \varepsilon^{N-1}\|\Phi\|_{\mathcal{V}} \int_{\mathbb{R}^{N-1} \backslash B\left(0, \alpha \varepsilon^{\beta-1}\right)} \frac{1}{|s|^{N+1}} d s=c \varepsilon^{N-1}\|\Phi\|_{\mathcal{V}}\left(\alpha^{-1} \varepsilon^{1-\beta}\right)^{2} \\
& \leq c \varepsilon^{N-1+2(1-\beta)}\|\Phi\|_{\mathcal{V}}
\end{aligned}
$$

(3) From (2.17), we obtain

$$
\left|C_{\varepsilon}(x)\right| \leq \varepsilon^{N-1} \int_{\mathbb{R}^{N-1}} \eta_{\varepsilon}(\varepsilon s)\left|\varphi\left(s, \varepsilon^{-1} \psi(\varepsilon s)\right)-\varphi(s, 0)\right| d s .
$$

The mean value inequality entails

$$
\left|\varphi\left(s, \varepsilon^{-1} \psi(\varepsilon s)\right)-\varphi(s, 0)\right| \leq\left|\varepsilon^{-1} \psi(\varepsilon s)\right| \sup _{|t| \leq \varepsilon^{-1}|\psi(\varepsilon s)|}\left|\frac{\partial \varphi}{\partial z_{N}}(s, t)\right| .
$$

From (2.32) and (2.35) we derive

$$
\left|\varphi\left(s, \varepsilon^{-1} \psi(\varepsilon s)\right)-\varphi(s, 0)\right| \leq c\|\Phi\|_{\mathcal{V} \varepsilon|s|^{2-N} .}
$$

Yet, (2.34) yields $|y(t)-x| \geq|t|$ for all $t \in \mathcal{O}$, hence

$$
\forall t \in \mathcal{O}, \quad|t| \geq 2 \rho \varepsilon^{\beta} \Longrightarrow \varepsilon^{-\beta}|y(t)-x| \geq 2 \rho \Longrightarrow \eta_{\varepsilon}(t)=0 .
$$

We conclude that

$$
\left|C_{\varepsilon}(x)\right| \leq c\|\Phi\|_{\mathcal{V} \varepsilon^{N}} \int_{B\left(0,2 \rho \varepsilon^{\beta-1}\right)}|s|^{2-N} d s=c\|\Phi\|_{\mathcal{V}} \varepsilon^{N+\beta-1} .
$$


(4) We get from (2.18)

$$
\left|D_{\varepsilon}(x)\right| \leq \varepsilon^{N-1} \int_{\mathbb{R}^{N-1}} \eta_{\varepsilon}(\varepsilon s)\left|\varphi\left(s, \varepsilon^{-1} \psi(\varepsilon s)\right)\right||\nabla \psi(\varepsilon s)| d s .
$$

Using that $|\nabla \psi(t)| \leq c|t|$ for all $t \in \mathcal{O}$ together with (2.28) for $n=N-1$, we obtain

$$
\left|D_{\varepsilon}(x)\right| \leq c\|\Phi\|_{\mathcal{V}} \varepsilon^{N} \int_{\mathbb{R}^{N-1}} \eta_{\varepsilon}(\varepsilon s)|s|^{2-N} d s .
$$

Using now (2.38), we arrive at

$$
\left|D_{\varepsilon}(x)\right| \leq c\|\Phi\|_{\mathcal{V}} \varepsilon^{N+\beta-1} .
$$

Gathering (2.22), (2.33), (2.37), (2.39) and (2.40), we finally obtain

$$
\left|T_{\varepsilon}(\Phi)-\tilde{T}_{\varepsilon}(\Phi)\right| \leq c\|\Phi\|_{\mathcal{V}} \varepsilon^{\alpha} \quad \forall \Phi \in \mathcal{C}_{0}^{\infty}(\mathbb{R}),
$$

for the exponent

$$
\begin{aligned}
\alpha & =2-N+\min ((1-\beta)(N+1), N-1+2(1-\beta), N+\beta-1) \\
& =\min (3-\beta(N+1), 3-2 \beta, 1+\beta)=\min (3-\beta(N+1), 1+\beta) .
\end{aligned}
$$

This value is maximized when $3-\beta(N+1)=1+\beta$, i.e., when $\beta=2 /(N+2)$. This corresponds to $\alpha=(N+4) /(N+2)$.

2.4. Extension to a function $\Phi \in \mathcal{V}$. We shall now extend (2.41) to an arbitrary function $\Phi \in \mathcal{V}$. The expression of the integral in (2.2) in spherical coordinates reads

$$
T_{\varepsilon}(\Phi)=\varepsilon^{-N} \int_{0}^{\infty} \int_{\mathcal{S}_{N-1}} \Phi(r) r^{2}\left|\hat{u}\left(\varepsilon^{-1} r v\right)\right|^{2} r^{N-1} d \sigma(v) d r=\int_{0}^{\infty} \Phi(r) r^{2} w_{\varepsilon}(r) d r,
$$

with

$$
w_{\varepsilon}(r)=\varepsilon^{-N} r^{N-1} \int_{\mathcal{S}_{N-1}}\left|\hat{u}\left(\varepsilon^{-1} r v\right)\right|^{2} d v,
$$

and $\mathcal{S}_{N-1}$ the unit sphere of $\mathbb{R}^{N-1}$. Note that, as $\hat{u} \in L^{2}\left(\mathbb{R}^{N}\right)$, we have $w_{\varepsilon} \in L^{1}\left(\mathbb{R}_{+}\right)$. We also write

$$
\tilde{T}_{\varepsilon}(\Phi)=\int_{0}^{\infty} \Phi(r) r^{2} \tilde{w}_{\varepsilon}(r) d r, \quad \text { with } \quad \tilde{w}_{\varepsilon}(r)=\frac{\varepsilon}{\pi} \frac{\operatorname{Per}(\Omega)}{r^{2}} .
$$

Therefore we have

$$
T_{\varepsilon}(\Phi)-\tilde{T}_{\varepsilon}(\Phi)=\int_{0}^{\infty} \Phi(r) r^{2}\left[w_{\varepsilon}(r)-\tilde{w}_{\varepsilon}(r)\right] d r
$$

From (2.41) and the above equality we derive that

$$
\left|\int_{0}^{\infty} \Phi(r) r^{2}\left[w_{\varepsilon}(r)-\tilde{w}_{\varepsilon}(r)\right] d r\right| \leq c \varepsilon^{\alpha}\|\Phi\|_{\mathcal{V}} \quad \forall \Phi \in \mathcal{C}_{0}^{\infty}(\mathbb{R}) .
$$

We choose now an arbitrary function $\Phi \in \mathcal{V}$, and construct the sequence of auxiliary functions

$$
\Phi_{n}(r)=\Phi(r) \eta\left(\frac{r}{n}\right)
$$

where $\eta \in \mathcal{C}^{\infty}(\mathbb{R})$ is such that $\eta(r)=1$ if $|r| \leq 1,0 \leq \eta(r) \leq 1$ if $1 \leq|r| \leq 2$, and $\eta(r)=0$ if $|r| \geq 2$. The differentiation of (2.43) at the order $k$ by the Leibniz formula and a reordering gives

$$
\forall n \in \mathbb{N}^{\star}, \forall r \in \mathbb{R}, \quad r^{k-2}\left(1+r^{2}\right)^{2} \Phi_{n}^{(k)}(r)=\sum_{p=0}^{k}\left(\begin{array}{l}
k \\
p
\end{array}\right) r^{p-2}\left(1+r^{2}\right)^{2} \Phi^{(p)}(r)\left(\frac{r}{n}\right)^{k-p} \eta^{k-p}\left(\frac{r}{n}\right) .
$$

For each $q \in \mathbb{N}$ the function $t \mapsto t^{q} \eta^{(q)}(t)$ belongs to $\mathcal{C}_{0}^{\infty}(\mathbb{R})$, hence it is bounded. This entails

$$
\forall n \in \mathbb{N}^{\star}, \quad\left\|\Phi_{n}\right\|_{\mathcal{V}_{k}} \leq c_{k} \sum_{p=0}^{k}\|\Phi\|_{\mathcal{V}_{p}}
$$

for some constants $c_{k}$ independent of $n$, and subsequently

$$
\forall n \in \mathbb{N}^{\star}, \quad\left\|\Phi_{n}\right\|_{\mathcal{V}} \leq c\|\Phi\|_{\mathcal{V}} .
$$


Applying (2.42) to the function $\Phi_{n}$ and using (2.44), it follows that

$$
\forall n \in \mathbb{N}^{\star}, \quad\left|\int_{0}^{\infty} \eta\left(\frac{r}{n}\right) \Phi(r) r^{2}\left[w_{\varepsilon}(r)-\tilde{w}_{\varepsilon}(r)\right] d r\right| \leq c \varepsilon^{\alpha}\|\Phi\|_{\mathcal{V}} .
$$

By Lebesgue's dominated convergence theorem, we can pass to the limit and find

$$
\left|\int_{0}^{\infty} \Phi(r) r^{2}\left[w_{\varepsilon}(r)-\tilde{w}_{\varepsilon}(r)\right] d r\right| \leq c \varepsilon^{\alpha}\|\Phi\|_{\mathcal{V}}
$$

that is,

$$
\left|T_{\varepsilon}(\Phi)-\tilde{T}_{\varepsilon}(\Phi)\right| \leq c \varepsilon^{\alpha}\|\Phi\|_{\mathcal{V}} .
$$

The proof of Theorem 2.1 is now complete.

\section{EXTENSION TO A BOUNDARY VALUE PROBLEM}

We assume now that $\Omega \subset \subset D$, where $D$ is a bounded Lipschitz domain of $\mathbb{R}^{N}$ and $\Omega$ has a $\mathcal{C}^{2}$ boundary. We consider the problem: find $v_{\varepsilon} \in H^{1}(D)$ such that

$$
\begin{cases}-\varepsilon^{2} \Delta v_{\varepsilon}+v_{\varepsilon}=u & \text { in } D, \\ \partial_{n} v_{\varepsilon}=0 & \text { on } \partial D,\end{cases}
$$

with $u$ the characteristic function of $\Omega$ in $D$, and set

$$
E_{\varepsilon}(\Omega)=\left\|u-v_{\varepsilon}\right\|_{L^{2}(D)}^{2} .
$$

Note that we have restricted ourselves to the case $m=1$ merely for simplicity. We shall show that $E_{\varepsilon}(\Omega)$ obeys the same first order asymptotic expansion as in the unbounded case.

Theorem 3.1. The following asymptotic expansion holds when $\varepsilon$ goes to zero:

$$
E_{\varepsilon}(\Omega)=\frac{\varepsilon}{4} \operatorname{Per}(\Omega)+O\left(\varepsilon^{\frac{N+4}{N+2}}\right) .
$$

Proof. We make the splitting $v_{\varepsilon}=u_{\varepsilon}+e_{\varepsilon}$ with $u_{\varepsilon} \in H^{1}\left(\mathbb{R}^{N}\right)$ and $e_{\varepsilon} \in H^{1}(D)$ respectively solutions of

$$
-\varepsilon^{2} \Delta u_{\varepsilon}+u_{\varepsilon}=u \text { in } \mathbb{R}^{N}, \quad\left\{\begin{array}{lll}
-\varepsilon^{2} \Delta e_{\varepsilon}+e_{\varepsilon}=0 & \text { in } & D, \\
\partial_{n} e_{\varepsilon}=-\partial_{n} u_{\varepsilon} & \text { on } & \partial D .
\end{array}\right.
$$

Here, $u$ is extended by zero outside $D$. We introduce the rescaled function $U_{\varepsilon}(x):=u_{\varepsilon}(\varepsilon x)$, which solves

Thus we can write for all $x \in \mathbb{R}^{N}$

$$
-\Delta U_{\varepsilon}+U_{\varepsilon}=u(\varepsilon x) \text { in } \mathbb{R}^{N} .
$$

$$
U_{\varepsilon}(x)=\int_{\mathbb{R}^{N}} u(\varepsilon y) \Gamma(x-y) d y,
$$

where $\Gamma$ is the fundamental solution of the operator $-\Delta+I$ in $\mathbb{R}^{N}$. By change of variable we obtain

$$
u_{\varepsilon}(x)=\varepsilon^{-N} \int_{\Omega} \Gamma\left(\frac{x-z}{\varepsilon}\right) d z .
$$

Assume now that $\operatorname{dist}(x, \Omega) \geq \rho>0$. By Fourier transform, we can easily show that $|\Gamma(x)|=O\left(|x|^{-p}\right)$ for all $p>0$. This implies

We arrive at

$$
\forall z \in \Omega, \quad\left|\Gamma\left(\frac{x-z}{\varepsilon}\right)\right| \leq c\left(\frac{\varepsilon}{\rho}\right)^{p} .
$$

$$
\left|u_{\varepsilon}(x)\right| \leq c|\Omega| \rho^{-p} \varepsilon^{p-N} .
$$

Similar estimates hold for $\left|\nabla u_{\varepsilon}(x)\right|$ and $\left|\Delta u_{\varepsilon}(x)\right|$, which provides, for any $k>0$,

$$
\left\|u_{\varepsilon}\right\|_{H^{1}\left(\mathbb{R}^{N} \backslash D\right)} \leq c \varepsilon^{k}, \quad\left\|\partial_{n} u_{\varepsilon}\right\|_{H^{-1 / 2}(\partial D)} \leq c \varepsilon^{k} .
$$

Now, the variational formulation of (3.4) yields

from which we deduce

$$
\int_{D}\left(\varepsilon^{2}\left|\nabla e_{\varepsilon}\right|^{2}+\left|e_{\varepsilon}\right|^{2}\right) d x=-\int_{\partial D} \partial_{n} u_{\varepsilon} e_{\varepsilon} d x,
$$

$$
\varepsilon^{2}\left\|e_{\varepsilon}\right\|_{H^{1}(D)} \leq c\left\|\partial_{n} u_{\varepsilon}\right\|_{H^{-1 / 2}(\partial D)} \leq c \varepsilon^{k} .
$$


Then we write

$$
\begin{aligned}
E_{\varepsilon}(\Omega) & =\left\|u-u_{\varepsilon}\right\|_{L^{2}(D)}^{2}-2 \int_{D} e_{\varepsilon}\left(u-u_{\varepsilon}\right) d x+\left\|e_{\varepsilon}\right\|_{L^{2}(D)}^{2} \\
& =\left\|u-u_{\varepsilon}\right\|_{L^{2}\left(\mathbb{R}^{N}\right)}^{2}-\left\|u_{\varepsilon}\right\|_{L^{2}\left(\mathbb{R}^{N} \backslash D\right)}^{2}-2 \int_{D} e_{\varepsilon}\left(u-u_{\varepsilon}\right) d x+\left\|e_{\varepsilon}\right\|_{L^{2}(D)}^{2} .
\end{aligned}
$$

By Theorem 1.1 we have

$$
\left\|u-u_{\varepsilon}\right\|_{L^{2}\left(\mathbb{R}^{N}\right)}^{2}=\frac{\varepsilon}{4} \operatorname{Per}(\Omega)+O\left(\varepsilon^{\frac{N+4}{N+2}}\right) .
$$

Combining (3.8), (3.5), (3.6) and (3.9), using the Cauchy-Schwarz inequality and choosing $k$ sufficiently large yields (3.3).

It is also of interest for the applications to study domains of the form $D \backslash \bar{\Omega}$, where $\Omega$ is defined as before. The peculiarity of this set is to touch the external boundary $\partial D$. The corresponding functional $E_{\varepsilon}(D \backslash \bar{\Omega})$ is defined by (3.1) and (3.2), with $u$ the characteristic function of $D \backslash \bar{\Omega}$. It turns out that the previous asymptotic expansion remains valid in this case, as stated in the following corollary.

Corollary 3.2. The following asymptotic expansion holds when $\varepsilon$ goes to zero:

$$
E_{\varepsilon}(D \backslash \bar{\Omega})=\frac{\varepsilon}{4} \operatorname{Per}(\Omega)+O\left(\varepsilon^{\frac{N+4}{N+2}}\right) .
$$

Proof. We have by definition

$$
E_{\varepsilon}(D \backslash \bar{\Omega})=\left\|u^{D \backslash \bar{\Omega}}-v_{\varepsilon}^{D \backslash \bar{\Omega}}\right\|_{L^{2}(D)}^{2},
$$

where $u^{D \backslash \bar{\Omega}}$ is the characteristic function of $D \backslash \bar{\Omega}$ and $v_{\varepsilon}^{D \backslash \bar{\Omega}}$ solves

$$
\left\{\begin{array}{lll}
-\varepsilon^{2} \Delta v_{\varepsilon}^{D \backslash \bar{\Omega}}+v_{\varepsilon}^{D \backslash \bar{\Omega}}=u^{D \backslash \bar{\Omega}} & \text { in } & D, \\
\partial_{n} v_{\varepsilon}^{D \backslash \bar{\Omega}}=0 & \text { on } & \partial D .
\end{array}\right.
$$

Since $u^{D \backslash \bar{\Omega}}=1-u^{\Omega}$ (almost everywhere), with $u^{\Omega}$ the characteristic function of $\Omega$, and, by uniqueness, $v_{\varepsilon}^{D \backslash \bar{\Omega}}=1-v_{\varepsilon}^{\Omega}$, with $v_{\varepsilon}^{\Omega}$ the solution of (3.1) for $u=u^{\Omega}$, we derive

$$
E_{\varepsilon}(D \backslash \bar{\Omega})=\left\|u^{\Omega}-v_{\varepsilon}^{\Omega}\right\|_{L^{2}(D)}^{2}=E_{\varepsilon}(\Omega) .
$$

Then we apply Theorem 3.1.

Note that, in this case, it is still the perimeter of $\Omega$ which is involved, not that of $D \backslash \bar{\Omega}$. In fact, this corresponds to the relative perimeter of $D \backslash \bar{\Omega}$ in $D$, namely $\sigma(\partial(D \backslash \bar{\Omega}) \cap D)$, see, e.g., [13].

\section{Topological SENSitivity of THE REgularized PERIMETER}

We place ourselves in the context if Section 3, i.e., we consider a bounded Lipschitz domain $D$ of $\mathbb{R}^{N}$ which will serve as "hold all". In this section we assume that $\varepsilon>0$ is fixed. For all $u \in L^{2}(D)$, we denote by $L_{\varepsilon} u$ the solution $v_{\varepsilon}$ of $(3.1)$, and we set

$$
P_{\varepsilon}(u)=\int_{D} L_{\varepsilon} u\left(L_{\varepsilon} u-2 u\right) d x .
$$

The functional $E_{\varepsilon}(\Omega)$ introduced in the previous section is defined for any measurable subset $\Omega$ of $D$ (it is not needed here to assume further regularity neither that $\Omega \subset \subset D)$ by $E_{\varepsilon}(\Omega)=\left\|L_{\varepsilon} \chi_{\Omega}-\chi_{\Omega}\right\|_{L^{2}(D)}^{2}$, with $\chi_{\Omega}$ the characteristic function of $\Omega$ in $D$. Then the regularized perimeter $\operatorname{Per}_{\varepsilon}(\Omega)$ defined by (1.2) satisfies

$$
\operatorname{Per}_{\varepsilon}(\Omega)=\frac{4}{\varepsilon}\left\|L_{\varepsilon} \chi_{\Omega}-\chi_{\Omega}\right\|_{L^{2}(D)}^{2}=\frac{4}{\varepsilon}\left[P_{\varepsilon}\left(\chi_{\Omega}\right)+|\Omega|\right],
$$

where $|\Omega|$ is the $N$-dimensional Lebesgue measure of $\Omega$.

Lemma 4.1. For any $q \in] 1,2]$ if $N=2, q \in[6 / 5,2]$ if $N=3$, the functional $u \in L^{q}(D) \mapsto P_{\varepsilon}(u)$ is of class $\mathcal{C}^{\infty}$ in the sense of Fréchet. Its derivative in the direction $h \in L^{q}(D)$ is given by

$$
D P_{\varepsilon}(u) h=2 \int_{D}\left(p_{\varepsilon}-v_{\varepsilon}\right) h d x,
$$


where $v_{\varepsilon}=L_{\varepsilon} u$ is the direct state and $p_{\varepsilon}$ is an adjoint state solution of

$$
\left\{\begin{array}{lll}
-\varepsilon^{2} \Delta p_{\varepsilon}+p_{\varepsilon}=v_{\varepsilon}-u & \text { in } & D \\
\partial_{n} p_{\varepsilon}=0 & \text { on } & \partial D
\end{array}\right.
$$

Proof. First, by application of the Lax-Milgram theorem, the map $L_{\varepsilon}:\left(H^{1}(D)\right)^{\prime} \rightarrow H^{1}(D)$ is linear and continuous. In addition, we have the continuous imbeddings $H^{1}(D) \hookrightarrow L^{q^{\prime}}(D)$ and $L^{q}(D) \hookrightarrow$ $\left(H^{1}(D)\right)^{\prime}$, where $q^{\prime}$ is such that $1 / q+1 / q^{\prime}=1$. Thus the map $u \in L^{q}(D) \mapsto P_{\varepsilon}(u)$ is of class $\mathcal{C}^{\infty}$ by composition. The standard rules of differential calculus provide

$$
D P_{\varepsilon}(u) h=\int_{D}\left[L_{\varepsilon} h\left(L_{\varepsilon} u-2 u\right)+L_{\varepsilon} u\left(L_{\varepsilon} h-2 h\right)\right] d x .
$$

A rearrangement and the replacement of $L_{\varepsilon} u$ by $v_{\varepsilon}$ yields

$$
D P_{\varepsilon}(u) h=2 \int_{D}\left[\left(v_{\varepsilon}-u\right) L_{\varepsilon} h-v_{\varepsilon} h\right] d x .
$$

Since the operator $L_{\varepsilon}: L^{q}(D) \rightarrow L^{q^{\prime}}(D)$ is self-adjoint, we can also write

$$
D P_{\varepsilon}(u) h=2 \int_{D}\left[L_{\varepsilon}\left(v_{\varepsilon}-u\right) h-v_{\varepsilon} h\right] d x .
$$

The definition of the adjoint state as $p_{\varepsilon}=L_{\varepsilon}\left(v_{\varepsilon}-u\right)$ leads to (4.2).

Theorem 4.2. Let $\Omega$ be a measurable subset of $D$ and $v_{\varepsilon}, p_{\varepsilon}$ be the direct and adjoint states, respectively, solutions of

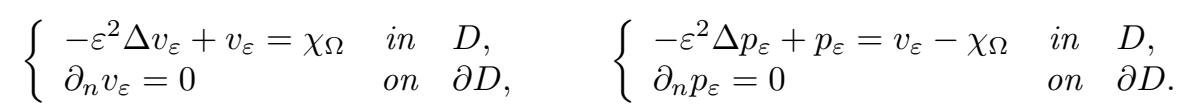

For any $q$ chosen as in Lemma 4.1 and any measurable subset $\tilde{\Omega}$ of $D$, we have

$$
\operatorname{Per}_{\varepsilon}(\tilde{\Omega})-\operatorname{Per}_{\varepsilon}(\Omega)=\int_{D} \operatorname{Per}_{\varepsilon}^{\prime}(\Omega)\left(\chi_{\tilde{\Omega}}-\chi_{\Omega}\right) d x+O\left(\left\|\chi_{\tilde{\Omega}}-\chi_{\Omega}\right\|_{L^{1}(D)}^{2 / q}\right),
$$

with the function $\operatorname{Per}_{\varepsilon}^{\prime}(\Omega)$ given by

$$
\operatorname{Per}_{\varepsilon}^{\prime}(\Omega)=\frac{4}{\varepsilon}\left[1+2\left(p_{\varepsilon}-v_{\varepsilon}\right)\right] .
$$

Proof. We get from (4.1)

$$
\operatorname{Per}_{\varepsilon}(\tilde{\Omega})-\operatorname{Per}_{\varepsilon}(\Omega)=\frac{4}{\varepsilon}\left[P_{\varepsilon}\left(\chi_{\tilde{\Omega}}\right)-P_{\varepsilon}\left(\chi_{\Omega}\right)+|\tilde{\Omega}|-|\Omega|\right] .
$$

A Taylor-Lagrange expansion of $P_{\varepsilon}$ yields

$$
\operatorname{Per}_{\varepsilon}(\tilde{\Omega})-\operatorname{Per}_{\varepsilon}(\Omega)=\frac{4}{\varepsilon}\left[D P_{\varepsilon}\left(\chi_{\Omega}\right)\left(\chi_{\tilde{\Omega}}-\chi_{\Omega}\right)+O\left(\left\|\chi_{\tilde{\Omega}}-\chi_{\Omega}\right\|_{L^{q}(D)}^{2}\right)+|\tilde{\Omega}|-|\Omega|\right] .
$$

Then (4.2) entails

$$
\operatorname{Per}_{\varepsilon}(\tilde{\Omega})-\operatorname{Per}_{\varepsilon}(\Omega)=\frac{4}{\varepsilon}\left[\int_{D} 2\left(p_{\varepsilon}-v_{\varepsilon}\right)\left(\chi_{\tilde{\Omega}}-\chi_{\Omega}\right) d x+O\left(\left\|\chi_{\tilde{\Omega}}-\chi_{\Omega}\right\|_{L^{1}(D)}^{2 / q}\right)+\int_{D}\left(\chi_{\tilde{\Omega}}-\chi_{\Omega}\right) d x\right] .
$$

A rearrangement completes the proof.

Remark 4.3. Suppose that $\chi_{\tilde{\Omega}}-\chi_{\Omega}=\chi_{B(z, \rho)}$ for some $z \in D$ and $\rho>0$. By elliptic regularity, $\operatorname{Per}_{\varepsilon}^{\prime}(\Omega)$ is continuous in the vicinity of $z$, hence, as $\rho \rightarrow 0$, the first term at the right hand side of (4.4) is equivalent to $\operatorname{Per}_{\varepsilon}^{\prime}(\Omega)(z)|B(z, \rho)|$. The second term is a $O\left(|B(z, \rho)|^{2 / q}\right)$ which, by choosing $q<2$, is of higher order than the first one. The function $\operatorname{Per}_{\varepsilon}^{\prime}(\Omega)$ can therefore be identified as the topological derivative $[4,10,17,18,19]$ of the shape functional $\operatorname{Per}_{\varepsilon}$ evaluated at $\Omega$. 


\section{Application to topology optimization}

Given a function $w \in L^{2}(D)$ and a real parameter $\alpha$, we consider the model problem

$$
\min _{\Omega \subset D} J(\Omega):=\int_{\Omega} w d x+\alpha \operatorname{Per}(\Omega) .
$$

Above, $\operatorname{Per}(\Omega)$ stands for the relative perimeter of $\Omega$ in $D$, whose definition can be extended to any measurable subset of $D[8,13]$. When $\alpha>0$, the existence of a minimizer is ensured, see, e.g., Theorem 1.4.5 of [8] or Theorem 4.1.4 of [13]. For any $\varepsilon>0$, we define the approximated problem

$$
\min _{\Omega \subset D} J_{\varepsilon}(\Omega):=\int_{\Omega} w d x+\alpha \operatorname{Per}_{\varepsilon}(\Omega)
$$

We use a continuation method described below.

Algorithm 5.1. (1) Define an initial domain $\Omega_{0}$, and a decreasing sequence $\left(\varepsilon_{n}\right)_{n \in \mathbb{N}}$ of positive numbers such that $\lim _{n \rightarrow \infty} \varepsilon_{n}=0$. Set $n=0$.

(2) Solve (5.1) with $\varepsilon=\varepsilon_{n}$ and the initial guess $\Omega_{n}$. Call $\Omega_{n+1}$ the obtained solution.

(3) Increment $n \leftarrow n+1$ and goto step (2).

To solve (5.1), we use the algorithm introduced in [6] and analyzed in [5]. We recall its main features. First, we need the topological derivative of the functional $J_{\varepsilon}$. It can be straightforwardly deduced from Theorem 4.2, which provides the topological asymptotic expansion

$$
J_{\varepsilon}(\tilde{\Omega})-J_{\varepsilon}(\Omega)=\int_{D} J_{\varepsilon}^{\prime}(\Omega)\left(\chi_{\tilde{\Omega}}-\chi_{\Omega}\right) d x+o\left(\left\|\chi_{\tilde{\Omega}}-\chi_{\Omega}\right\|_{L^{1}(D)}\right)
$$

with

$$
J_{\varepsilon}^{\prime}(\Omega)=w+\alpha \operatorname{Per}_{\varepsilon}^{\prime}(\Omega) .
$$

From (5.2) we deduce the following necessary optimality conditions:

$$
\begin{array}{ll}
J_{\varepsilon}^{\prime}(\Omega) \leq 0 & \text { a.e. in } \quad \Omega, \\
J_{\varepsilon}^{\prime}(\Omega) \geq 0 & \text { a.e. in } \quad D \backslash \bar{\Omega} .
\end{array}
$$

To solve these conditions, we represent every domain $\Omega \subset D$ by a so-called level-set function $\psi: D \rightarrow \mathbb{R}$ constructed so that

$$
\Omega=\Omega(\psi):=\{x \in D, \psi(x)<0\} .
$$

We equip the set of real valued functions defined on $D$ with the equivalence relation:

$$
\psi_{1} \sim \psi_{2} \Longleftrightarrow \exists \mu>0, \psi_{1}=\mu \psi_{2}
$$

Therefore, the conditions (5.3) will be satisfied by the domain $\Omega(\psi)$ whenever

$$
J_{\varepsilon}^{\prime}(\Omega(\psi)) \sim \psi
$$

We solve this equation by the fixed point iteration with relaxation applied to the equivalence classes. It turns out to be convenient to handle representatives on the unit sphere $\mathcal{S}$ of some Hilbert space $\mathcal{H}$ of functions on $D$, for instance $\mathcal{H}=L^{2}(D)$. This leads to the following algorithm.

Algorithm 5.2. (1) Choose an initial function $\psi_{0} \in \mathcal{S}$. Set $k=0$.

(2) Determine $\psi_{k+1} \in \mathcal{S}$ as

$$
\psi_{k+1} \sim\left(1-\lambda_{k}\right) \psi_{k}+\lambda_{k} J_{\varepsilon}^{\prime}\left(\Omega\left(\psi_{k}\right)\right),
$$

with $\left.\left.\lambda_{k} \in\right] 0,1\right]$ chosen so that

$$
J_{\varepsilon}\left(\Omega\left(\psi_{k+1}\right)\right) \leq J_{\varepsilon}\left(\Omega\left(\psi_{k}\right)\right) .
$$

(3) Increment $n \leftarrow n+1$ and goto step (2).

The interest of the Hilbertian norm is that (5.4) can be reformulated as

with the angle

$$
\psi_{k+1}=\frac{1}{\sin \theta_{k}}\left[\sin \left(\left(1-\tau_{k}\right) \theta_{k}\right) \psi_{k}+\sin \left(\tau_{k} \theta_{k}\right) \frac{J_{\varepsilon}^{\prime}\left(\Omega\left(\psi_{k}\right)\right)}{\left\|J_{\varepsilon}^{\prime}\left(\Omega\left(\psi_{k}\right)\right)\right\|_{\mathcal{H}}}\right],
$$

$$
\theta_{k}=\arccos \left\langle\psi_{k}, \frac{J_{\varepsilon}^{\prime}\left(\Omega\left(\psi_{k}\right)\right)}{\left\|J_{\varepsilon}^{\prime}\left(\Omega\left(\psi_{k}\right)\right)\right\|_{\mathcal{H}}}\right\rangle_{\mathcal{H}},
$$



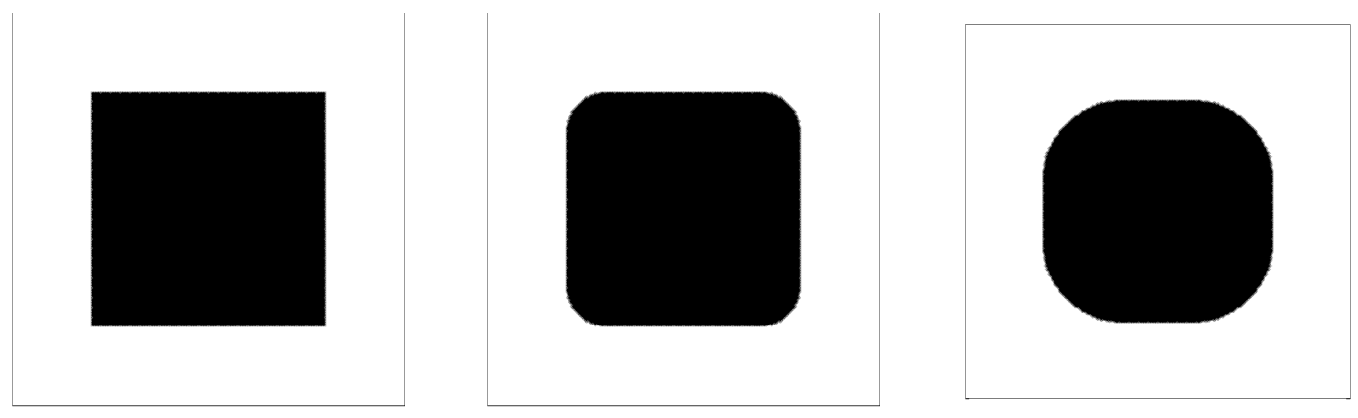

Figure 1. Example 1: $\alpha=0$ (left), $\alpha=0.1$ (middle), $\alpha=0.2$ (right).
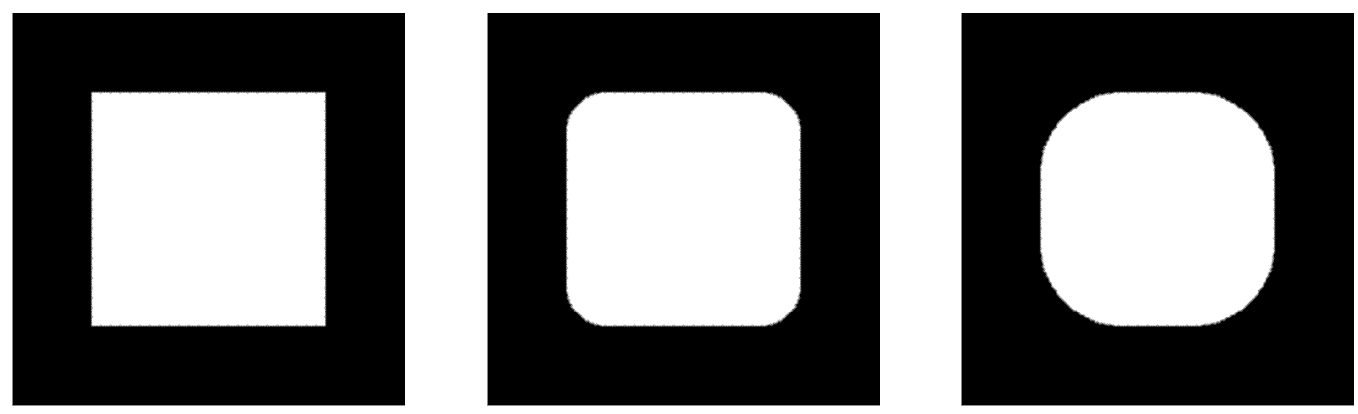

Figure 2. Example 2: $\alpha=0$ (left), $\alpha=0.1$ (middle), $\alpha=0.2$ (right).

and $\left.\left.\tau_{k} \in\right] 0,1\right]$ acting as stepsize in place of $\lambda_{k}$. In the implementation, $\tau_{k}$ is determined by a line search of Armijo type (see [5]).

\section{NUMERICAL EXPERIMENTS}

In the following examples the spatial dimension is $N=2$. The hold all $D$ is the unit square $] 0,1[2$. We choose the full domain initialization $\Omega_{0}=D$, more precisely, $\psi_{0}=-1 /\|1\|_{\mathcal{H}}$ with $\mathcal{H}=L^{2}(D)$. The direct and adjoint problems are solved in Matlab by piecewise linear finite elements on a structured mesh with 51521 degrees of freedom. The sequence of regularization parameters is chosen as $\varepsilon_{n}=1 / 2^{n}$, and 15 iterations of Algorithm 5.1 are performed. Actually, we observe that almost no more evolution occurs when $\varepsilon_{n}$ becomes smaller than the mesh resolution. The stopping criterion of Algorithm 5.2 is $\theta_{k} \leq 0.1^{\circ}$. For each presented example, the computer time of the whole procedure is lower than 5 minutes on a standard PC.

6.1. Example 1. The function $w$ is chosen as

$$
w\left(x_{1}, x_{2}\right)=\left\{\begin{aligned}
-1 & \text { if } 0.2 \leq x_{1}, x_{2} \leq 0.8 \\
1 & \text { otherwise }
\end{aligned}\right.
$$

In Figure 1, we present the results obtained with the coefficients $\alpha=0, \alpha=0.1$ and $\alpha=0.2$. Of course, for $\alpha=0$, the optimal solution is the rectangle $] 0.2,0.8\left[{ }^{2}\right.$. For $\alpha>0$, the contribution of the perimeter is highlighted by the rounded corners.

6.2. Example 2. In order to demonstrate the ability of the algorithm to deal with topology changes and illustrate Corollary 3.2, we choose now

$$
w\left(x_{1}, x_{2}\right)=\left\{\begin{aligned}
1 & \text { if } 0.2 \leq x_{1}, x_{2} \leq 0.8 \\
-1 & \text { otherwise }
\end{aligned}\right.
$$

The results obtained for the same values of $\alpha$ as in Example 1 are shown in Figure 2. 

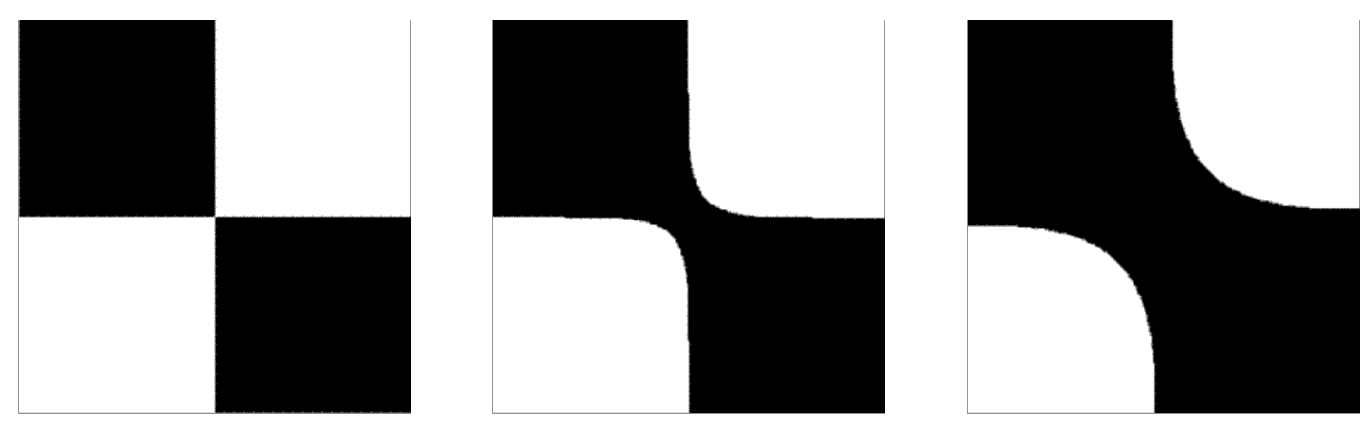

Figure 3. Example 3: $\alpha=0$ (left), $\alpha=0.01$ (middle), $\alpha=0.1$ (right).
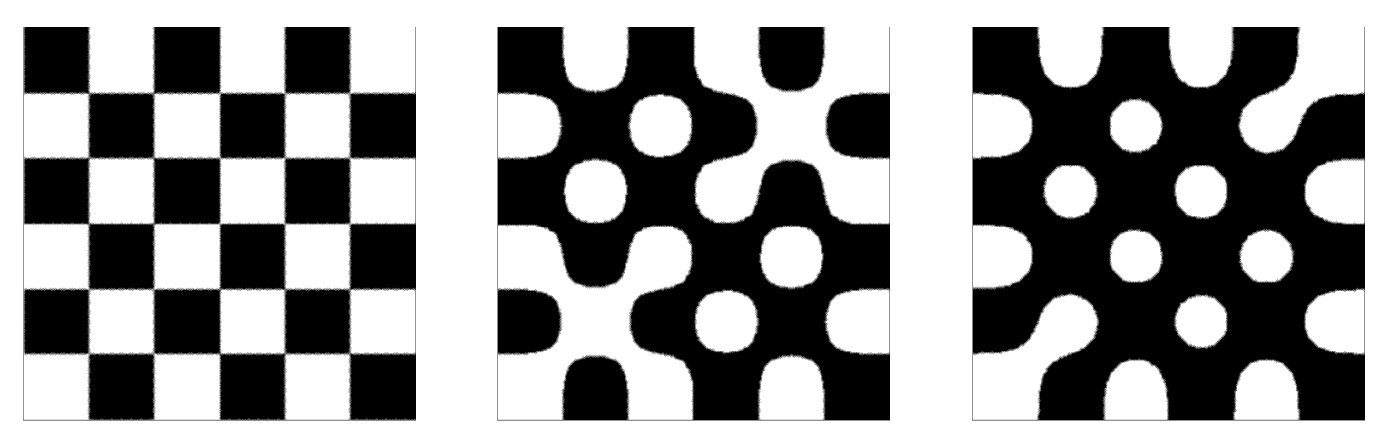

Figure 4. Example 4: $\alpha=0$ (left), $\alpha=0.01$ (middle), $\alpha=0.02$ (right).

6.3. Example 3. The purpose of this example is to show that the proposed algorithm can also be used when there exist junction points between $\partial \Omega$ and $\partial D$, although this case has not been treated in the theory. In fact, if the junctions occur at right angles, it is intuitively clear that, due to the Neumann boundary condition in (3.1), the functional $\operatorname{Per}_{\varepsilon}(\Omega)$ still approximates the relative perimeter of $\Omega$ in $D$, namely, $\sigma(\partial \Omega \cap D)$. We consider the function

$$
w\left(x_{1}, x_{2}\right)=\sin \left(2 \pi x_{1}\right) \sin \left(2 \pi x_{2}\right) .
$$

Figure 3 shows the results obtained with the coefficients $\alpha=0, \alpha=0.01$ and $\alpha=0.1$.

6.4. Example 4. In this last example we combine topology changes and junction of boundaries by choosing

$$
w\left(x_{1}, x_{2}\right)=\sin \left(\frac{2 \pi x_{1}}{3}\right) \sin \left(\frac{2 \pi x_{2}}{3}\right) .
$$

The results obtained with the coefficients $\alpha=0, \alpha=0.01$ and $\alpha=0.02$ are depicted in Figure 4 .

\section{ApPENDix}

In this appendix we prove the relation $(2.25)$ for every $p \in \mathbb{N}$. Obviously it is true for $p=0$. Suppose now that it is true for some $p \in \mathbb{N}$. The differentiation gives

$$
\begin{aligned}
& \frac{\partial^{q+1}}{\partial \zeta_{j}^{q+1}}\left(\frac{\Phi(|\zeta|)}{|\zeta|^{2}}\right)=-(2 q+2) \frac{\zeta_{j}}{|\zeta|^{2 q+4}} \sum_{p=0}^{q} \Phi^{(p)}(|\zeta|) P_{p, q}\left(|\zeta|, \zeta_{j}\right) \\
& \quad+\frac{1}{|\zeta|^{2 q+2}} \sum_{p=0}^{q}\left[\Phi^{(p+1)}(|\zeta|) \frac{\zeta_{j}}{|\zeta|} P_{p, q}\left(|\zeta|, \zeta_{j}\right)+\Phi^{(p)}(|\zeta|)\left(\partial_{1} P_{p, q}\left(|\zeta|, \zeta_{j}\right) \frac{\zeta_{j}}{|\zeta|}+\partial_{2} P_{p, q}\left(|\zeta|, \zeta_{j}\right)\right)\right] .
\end{aligned}
$$

For each $p \in\{0, \ldots, q\}$ we set

$$
\begin{gathered}
P_{p, q+1}^{1}\left(|\zeta|, \zeta_{j}\right)=-(2 q+2) \zeta_{j} P_{p, q}\left(|\zeta|, \zeta_{j}\right), \\
P_{p+1, q+1}^{2}\left(|\zeta|, \zeta_{j}\right)=\zeta_{j}|\zeta| P_{p, q}\left(|\zeta|, \zeta_{j}\right), \\
P_{p, q+1}^{3}\left(|\zeta|, \zeta_{j}\right)=\partial_{1} P_{p, q}\left(|\zeta|, \zeta_{j}\right) \zeta_{j}|\zeta|+\partial_{2} P_{p, q}\left(|\zeta|, \zeta_{j}\right)|\zeta|^{2} .
\end{gathered}
$$


We note that each polynomial $P_{\alpha, \beta}^{l}$ is homogeneous of degree $\alpha+\beta$. We obtain

$$
\begin{array}{r}
\frac{\partial^{q+1}}{\partial \zeta_{j}^{q+1}}\left(\frac{\Phi(|\zeta|)}{|\zeta|^{2}}\right)=\frac{1}{|\zeta|^{2 q+4}} \sum_{p=0}^{q}\left[\Phi^{(p)}(|\zeta|) P_{p, q+1}^{1}\left(|\zeta|, \zeta_{j}\right)+\Phi^{(p+1)}(|\zeta|) P_{p+1, q+1}^{2}\left(|\zeta|, \zeta_{j}\right)\right. \\
\left.+\Phi^{(p)}(|\zeta|) P_{p, q+1}^{3}\left(|\zeta|, \zeta_{j}\right)\right]
\end{array}
$$

A rearrangement entails

$$
\frac{\partial^{q+1}}{\partial \zeta_{j}^{q+1}}\left(\frac{\Phi(|\zeta|)}{|\zeta|^{2}}\right)=\frac{1}{|\zeta|^{2 q+4}} \sum_{p=0}^{q+1} \Phi^{(p)}(|\zeta|)\left[P_{p, q+1}^{1}\left(|\zeta|, \zeta_{j}\right)+P_{p, q+1}^{2}\left(|\zeta|, \zeta_{j}\right)+P_{p, q+1}^{3}\left(|\zeta|, \zeta_{j}\right)\right],
$$

where the undefined polynomials $P_{q+1, q+1}^{1}, P_{0, q+1}^{2}$ and $P_{q+1, q+1}^{3}$ have been set to zero. It suffices now to set $P_{p, q+1}=P_{p, q+1}^{1}+P_{p, q+1}^{2}+P_{p, q+1}^{3}$ to complete the proof.

Acknowledgements. I thank Frédéric Naud for interesting discussions, and the French National Center for Scientific Research (CNRS) for its support.

\section{REFERENCES}

[1] G. Allaire. Shape optimization by the homogenization method, volume 146 of Applied Mathematical Sciences. Springer-Verlag, New York, 2002.

[2] G. Allaire. Conception optimale de structures, volume 58 of Mathématiques \& Applications (Berlin) [Mathematics ES Applications]. Springer-Verlag, Berlin, 2007. With the collaboration of Marc Schoenauer (INRIA) in the writing of Chapter 8.

[3] L. Ambrosio and G. Buttazzo. An optimal design problem with perimeter penalization. Calc. Var. Partial Differential Equations, 1(1):55-69, 1993

[4] S. Amstutz. Sensitivity analysis with respect to a local perturbation of the material property. Asymptot. Anal., 49(1-2):87-108, 2006.

[5] S. Amstutz. Analysis of a level-set method for topology optimization. Optim. Methods Soft., To appear.

[6] S. Amstutz and H. Andrä. A new algorithm for topology optimization using a level-set method. J. Comput. Phys., 216(2):573-588, 2006

[7] H. Attouch, G. Buttazzo, and G. Michaille. Variational analysis in Sobolev and BV spaces, volume 6 of MPS/SIAM Series on Optimization. Society for Industrial and Applied Mathematics (SIAM), Philadelphia, PA, 2006. Applications to PDEs and optimization.

[8] D. Bucur and G. Buttazzo. Variational methods in shape optimization problems. Progress in Nonlinear Differential Equations and their Applications, 65. Birkhäuser Boston Inc., Boston, MA, 2005.

[9] A. Cherkaev. Variational methods for structural optimization, volume 140 of Applied Mathematical Sciences. Springer-Verlag, New York, 2000.

[10] S. Garreau, P. Guillaume, and M. Masmoudi. The topological asymptotic for PDE systems: the elasticity case. SIAM J. Control Optim., 39(6):1756-1778 (electronic), 2001.

[11] B. Hackl. Methods for reliable topology changes for perimeter-regularized geometric inverse problems. SIAM J. Numer. Anal., 45(5):2201-2227 (electronic), 2007.

[12] L. He and S. Osher. Solving the chan-vese model by a multiphase level set algorithm based on the topological derivative. In F. Sgallari, A. Murli, and N. Paragios, editors, Scale Space and Variational Methods in Computer Vision, Proceedings, Lecture Notes in Computer Science. 2007.

[13] A. Henrot and M. Pierre. Variation et optimisation de formes, volume 48 of Mathématiques \& Applications (Berlin) [Mathematics 83 Applications].

[14] M. Hintermüller and A. Laurain. Multiphase image segmentation and modulation recovery based on shape and topological sensitivity. J. Math. Imaging Vision, 35(1):1-22, 2009.

[15] L. Hörmander. The analysis of linear partial differential operators. I. Classics in Mathematics. Springer-Verlag, Berlin, 2003. Distribution theory and Fourier analysis, Reprint of the second (1990) edition [Springer, Berlin; MR1065993 (91m:35001a)].

[16] D. Mumford and J. Shah. Optimal approximations by piecewise smooth functions and associated variational problems. Comm. Pure Appl. Math., 42(5):577-685, 1989.

[17] S. A. Nazarov and J. Sokołowski. Asymptotic analysis of shape functionals. J. Math. Pures Appl. (9), 82(2):125-196, 2003.

[18] A. A. Novotny, R. A. Feijóo, E. Taroco, and C. Padra. Topological sensitivity analysis. Comput. Methods Appl. Mech. Engrg., 192(7-8):803-829, 2003.

[19] J. Sokołowski and A. Żochowski. On the topological derivative in shape optimization. SIAM J. Control Optim., 37(4):1251-1272 (electronic), 1999

Laboratoire de Mathématiques d’Avignon, Faculté des Sciences, 33 rue Louis Pasteur, 84000 Avignon, FRANCE. 
Institut de Mathématiques et de Modélisation de Montpellier, UMR CNRS \& Université de Montpellier 2, Place Eugène Bataillon, 34095 Montpellier Cedex, France.

E-mail address: samuel.amstutz@univ-avignon.fr 